\title{
Design and Optimization of Levenberg-Marquardt based Neural Network Classifier for EMG Signals to Identify Hand Motions
}

\author{
Muhammad Ibn Ibrahimy ${ }^{1}$, Md. Rezwanul Ahsan ${ }^{1,2}$, Othman Omran Khalifa ${ }^{1}$ \\ ${ }^{1}$ Dept. of ECE, Faculty of Engineering, International Islamic University Malaysia, 53100 Kuala Lumpur, Malaysia \\ ${ }^{2}$ Dept. of Electrical Electronic and System Engineering, Faculty of Engineering and Built Environment, \\ Universiti Kebangsaan Malaysia, 43600 UKM, Bangi, Malaysia. \\ Email: rezwanul.ahsan@yahoo.com,ibrahimy@iium.edu.my,khalifa@iium.edu.my
}

\begin{abstract}
This paper presents an application of artificial neural network for the classification of single channel EMG signal in the context of hand motion detection. Seven statistical input features that are extracted from the preprocessed single channel EMG signals recorded for four predefined hand motions have been used for neural network classifier. Different structures of neural network, based on the number of hidden neurons and two prominent training algorithms, have been considered in the research to find out their applicability for EMG signal classification. The classification performances are analyzed for different architectures of neural network by considering the number of input features, number of hidden neurons, learning algorithms, correlation between network outputs and targets, and mean square error. Between the Levenberg-Marquardt and scaled conjugate gradient learning algorithms, the aforesaid algorithm shows better classification performance. The outcomes of the research show that the optimal design of Levenberg-Marquardt based neural network classifier can perform well with an average classification success rate of 88.4\%. A comparison of results has also been presented to validate the effectiveness of the designed neural network classifier to discriminate EMG signals.
\end{abstract}

Keywords: Electromyography, neural network, back-propagation, Levenberg-Marquardt algorithm, EMG signal classifier

\section{INTRODUCTION}

$\mathrm{W}$ ITH THE advancement of computer centered technology, electromyography (EMG) signal finds its utilization in a variety of application areas. These may include analyzing of neuromuscular difficulties, controlling prosthetic/orthotic limbs, I/O for virtual-reality games, manipulation of physical exercise equipment, or development and controlling of human-computer interfaces (HCI) [1]. Over the span of the last 40 years, an extensive effort was put in by many researchers, and as a result improvement was observed in various directions of EMG signal monitoring, processing and controlling. However, in developing the EMG signal based controller, most of the researchers find the signal classification task the most challenging since it relies on certain functional requirements. It may be due to the enormous variations of EMG signal properties and morphology with differences in the signature characteristics subjected to age, muscle activity, motor unit pattern, skin-fat layer and gesture style. Furthermore, due to very sensitive nature of the EMG signal, it can easily exhibit interference by different types of noises. These noises may include inherent equipment and ambient noises, electromagnetic emissions, motion artifacts and cross-talk between nearby tissues [2]. In some cases, it is problematic to acquire the informative signal pattern from the residual weak muscle group of a disabled or amputee person. Even more complications may arise while dealing with the solution of multiclass classification problems [3].

EMG signals can be employed as an alternative input mechanism to control an external peripheral or device by identifying the motion commands. This is done by interpreting the signals originating from the body muscle and transforming it into desired control operations. To do so, some of the significant features are extracted out of the
EMG signals for each movement of hand muscles and then the EMG signal is classified by applying a proper discrimination technique based on extracted feature parameters. However, due to the intense complication, properties associated with the EMG signals result in getting a precise formulation for structural or mathematical model which can relate the signal to corresponding motion command. Artificial Neural Networks (ANN) have emerged as a significant and efficient tool for analyzing complex data and pattern classification. The ANNs are formed by mimicking the low-level tasks of biological neurons which make them particularly useful for recognizing and classifying complex patterns [4]. Some of the pioneer research work for EMG signal classification task can be mentioned herewith: integral absolute value (IAV) feature based feed-forward ANN [5], AR parameter based ANN [6], independent component analysis (ICA) based ANN [7], different multi-layer perceptron (MLP) based neural network [8]-[10], Hopfield and adaptive resonance theory (ART) based neural network, and later finite impulse response neural network (FIRNN) [11], and linear vector quantization (LVQ) type ANN [12]. It should be noted that different types of ANNs are available depending on their structure and training model. Hence, selection of proper ANN structure and training method is essential to perform a certain type of tasks efficiently. The review process found that most of the ANN based EMG signal classifiers suffer from a lack of information regarding network optimization. Moreover, insufficient articles have been found to discuss more precisely the improvement of the classification success by utilizing single channel EMG signals.

This research work mainly focused on designing an ANN classifier based back-propagation training algorithm and optimizing its structural design for the classification of 
EMG signals to gain acceptable accuracy by comparing with other related works. The Levenberg-Marquardt and scaled conjugate gradient based back-propagation training algorithms are used to train the network. These two training algorithms have been chosen since they provide faster training for solving pattern recognition problems using the numerical optimization technique [13]. Their classification performances with different network architecture are reported in the result section.

\section{SUBJECT \& METHODS}

ANN models are basically structured with many interconnected network elements which can develop pattern classification strategies based on a set of input/training data. The ANN models working in parallel provide higher computational performance than traditional classifiers which function sequentially. The EMG signals are obtained for different kinds of hand motions, which are further denoised and processed to extract the features. Extracted time and time-frequency based feature sets are used to train the neural network [14]. The block diagram of ANN based EMG signal classification is shown in Fig.1. The EMG signals for different hand motions are recorded and processed further for reducing the associated noise. Seven statistical time and time-frequency based features, namely Moving Average (MAV), RMS (Root Mean Square), VAR (Variance), SD (Standard Deviation), ZC (Zero-crossing), SSC (Slope Sign Change) and WL (Waveform Length) [15]-[18], are extracted from the EMG signals and used as inputs to the neural network. The subsequent sections explain the details of the design architecture and training of the ANN.

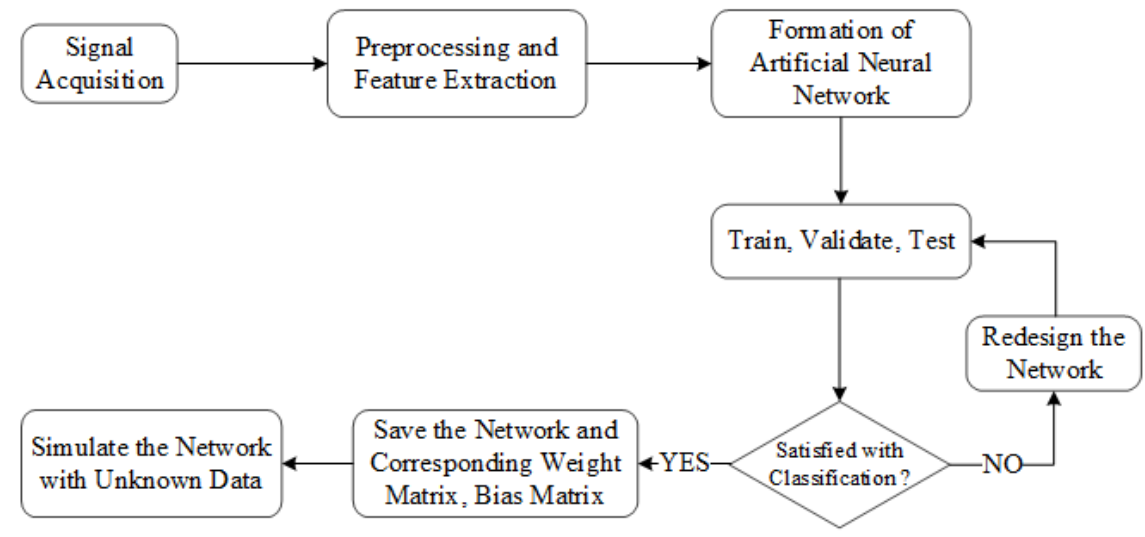

Fig.1. Block diagram for ANN based EMG signal classification

\section{A. Acquisition of EMG Signals}

Most of the previous research works utilized multichannel EMG signals to achieve better classification. Some of the previous studies [15], [19]-[21] present that it is beneficial to use EMG signals of multiple channels. However, though the use of increased numbers of channels will increase the average classification accuracy, a reduced efficiency may be observed for the numbers of channels greater than four [17]. On the other hand, some researchers are interested in considering the best and significant features other than using multichannel EMG signals or a combination of these approaches [22]. Placement of electrodes in different locations of muscle sites may help to achieve an improved classification rate despite the utilization of increased numbers of features.

For this work, the Surface EMG signals were acquired from 3 able-bodied persons aged 25-32. Before the signal acquisition process, each subject's consent has been taken by giving them the outline of the research work. The device used for the acquisition of single channel EMG signals was BIOPAC-MP100C data acquisition system (shown in Fig.2.) manufactured by BIOPAC Systems Inc. USA. It was equipped with data acquisition unit MP100A-CE, universal interface module UIM100C, electromyogram amplifier module EMG100C and acquisition software AcqKnowledge. v 3.1.9 [23]. The sampling frequency was
$1000 \mathrm{~Hz}$ and gain set to 1000 . The optimal position of the electrode placement has been determined by performing several trials of the acquisition experiment. As the hand movements are generated by the muscle contractions in forearm section, EMG signals are collected from the brachioradialis position and flexor carpi ulnaris muscle sites using differential electrodes [24]. Fig.3. shows the possible location of muscle site and placement of electrodes with the reference electrode near the wrist position. The muscle sites are selected carefully by properly placing the electrodes so that they provide strong activation pattern for EMG signals with minimal cross-talk [25]. A judicious placement of surface electrodes for different subjects is considered and a generous selection of the channel with more informative raw EMG signals is ensured. The EMG signals were collected for different voluntary movements of the subject's hand in four directions (Left, Right, Up and Down). From any reference point, movement of hand in a horizontal left direction is considered as Left, and Right is hand movement in horizontal right direction. Up is the upward movement of hand from the reference point and Down is downward movement. The average time required to perform each movement was around $500 \mathrm{~ms}$. Each EMG signal set has been collected for 70 seconds including 5 seconds rest at start and end of signal acquisition. It is considered to record around 50 to 55 actions in the time period of 60 seconds by 
practicing the speed of hand movement before starting the signal acquisition. The EMG signals are stored on a Windows XP based personal computer for post analysis and processing. MATLAB programming platform is used for coding the necessary modules and subroutines for EMG signal processing and ANN based classification.

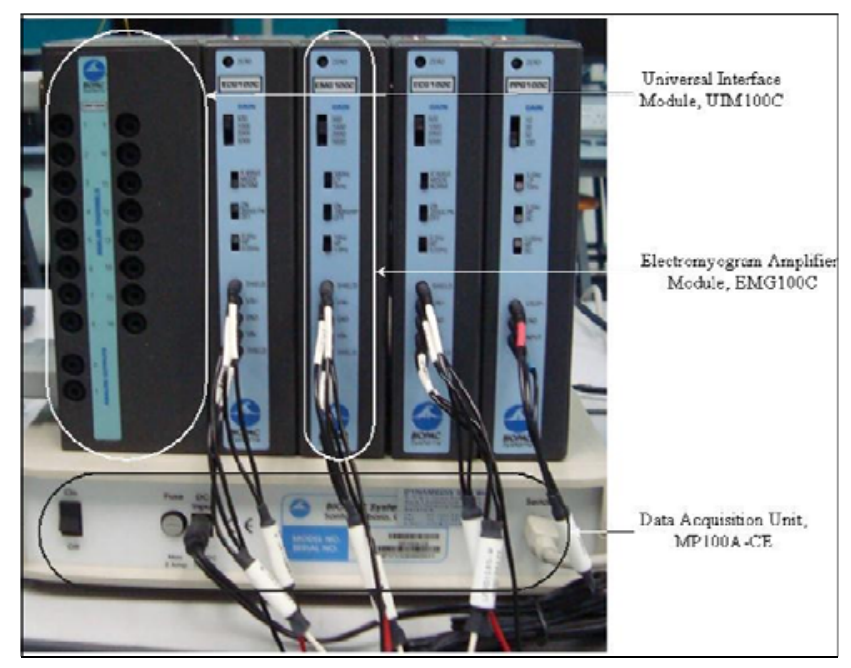

Fig.2. BIOPAC-MP100 data acquisition system (Biopac Systems Inc., USA)

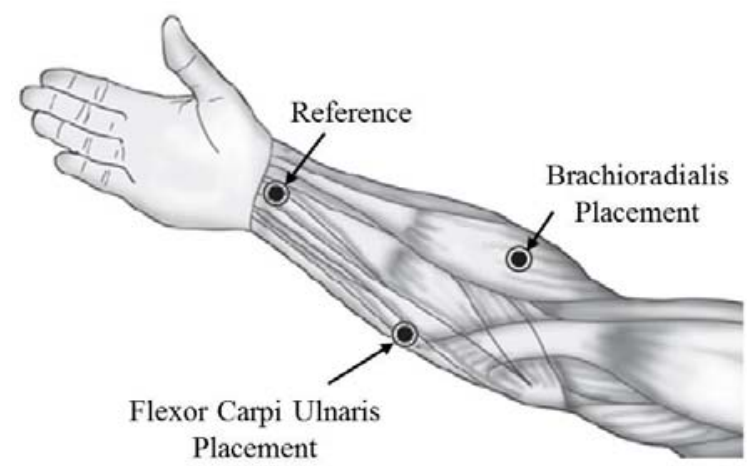

Fig.3. Muscle site and possible placement of electrodes

\section{B. Preprocessing and Feature Extraction}

Since the EMG signal is sensitive to external noise sources and artifacts, most noise sources can be classified as electrode noise, motion artifacts, power line noise, ambient noise, and inherent noise in electrical and electronic equipment. If these contaminated signals are used, it will yield undesirable, very poor classification. The first three types of noise can be removed by applying some typical filtering techniques like band-pass filter, band-stop filter or using good quality equipment with properly placed electrodes. However, sometimes it is difficult to eliminate the effect of other types of noises/artifacts if their frequencies overlap with the dominant frequency range of EMG signal [2]. A 6-th order Butterworth band-pass filter and cut-off frequency of $20-500 \mathrm{~Hz}$ is used to remove the first 3 types of noise. Since the dominant frequency range of the recorded signal is $70-300 \mathrm{~Hz}$, a notch filter with $3 \mathrm{db}$ gain and cut-off $49-51 \mathrm{~Hz}$ is applied to remove $50 \mathrm{~Hz}$ power line noise. Afterwards, the wavelet transform method is applied to EMG signal for denoising since it has various scales and resolutions to process signals and it can successfully localize both time and frequency components. Additionally, the wavelet technique provides good frequency resolution at high frequencies. So the noise components in the desired signal can be isolated while important high-frequency transients are preserved [17]. The collected EMG signal for 4 different hand movements (left, right, up, down) are segmented of 500 data points for each type of motion. A 4-level discrete wavelet transform (DWT) is used for the decompositions of EMG signal with Daubechies $(\mathrm{db} 2)$ mother wavelet function according to the previous research [26]. Later on, the features were extracted for each type of hand movement from the denoised EMG signals [23].

Because of complex signal pattern of EMG signals, it is essential to select significant features for efficient classification since it determines the success of the pattern classification system [11]. However, it is quite problematic to extract the best feature parameters from the EMG signals that can reflect the unique feature of the signal to the motion command perfectly. Hence, multiple feature sets are used as input to the EMG signal classification process. Some of the features are classified as the time domain, frequency domain, time-frequency domain, and time-scale domain; these feature types are successfully employed for EMG signal classification. In this research work, seven statistical property based time and time-frequency features, namely MAV, RMS, VAR, SD, ZC, SSC and WL, are used. For each type of hand motion, the above mentioned features are extracted from the segmented and denoised EMG signal and fed as input to the neural network based classifier. A total number of 204 sets of input vectors and target vectors are fed to the network for training purpose. The input feature vectors are normalized in the range of $[-1,+1]$ for the efficient and faster training of neural network. A sample input vector and its corresponding target vector are shown in Table.1.

Table 1. Sample Feature set as input and output vector for Artificial Neural Network

\begin{tabular}{|c|c|c|c|c|c|}
\hline \multirow{9}{*}{ 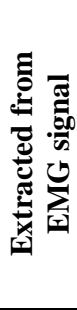 } & \multirow{2}{*}{ Features } & \multicolumn{4}{|c|}{ Movement type } \\
\hline & & Left & Right & $\mathrm{Up}$ & Down \\
\hline & MAV & 0.11862 & 0.10395 & 0.10554 & 0.07303 \\
\hline & RMS & 0.16918 & 0.14866 & 0.14803 & 0.09834 \\
\hline & VAR & 0.02862 & 0.02210 & 0.02191 & 0.00967 \\
\hline & $\mathrm{SD}$ & 0.16910 & 0.14861 & 0.14811 & 0.09841 \\
\hline & WL & 72.66327 & 65.94147 & 62.51984 & 45.28778 \\
\hline & $\mathrm{SSC}$ & 234 & 214 & 220 & 230 \\
\hline & $\mathrm{ZC}$ & 207 & 204 & 191 & 219 \\
\hline \multirow{8}{*}{ 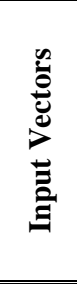 } & & \multicolumn{4}{|c|}{ After normalization in the range $\left[\begin{array}{ll}-1 & 1\end{array}\right]$} \\
\hline & MAV & 0.62547 & 0.36995 & 0.39767 & -0.16858 \\
\hline & RMS & 0.57261 & 0.33743 & 0.33016 & -0.23938 \\
\hline & VAR & 0.32669 & 0.01320 & 0.00415 & -0.58431 \\
\hline & $\mathrm{SD}$ & 0.57222 & 0.33665 & 0.33096 & -0.24050 \\
\hline & WL & 0.55323 & 0.37111 & 0.27841 & -0.18846 \\
\hline & $\mathrm{SSC}$ & 0.10811 & -0.43243 & -0.27027 & 0.00000 \\
\hline & $\mathrm{ZC}$ & -0.03226 & -0.12903 & -0.54839 & 0.35484 \\
\hline \multirow{5}{*}{ 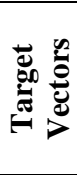 } & Set & \multicolumn{4}{|c|}{ Target Vector } \\
\hline & Left & 1 & 0 & 0 & 0 \\
\hline & Right & 0 & 1 & 0 & 0 \\
\hline & Up & 0 & 0 & 1 & 0 \\
\hline & Down & 0 & 0 & 0 & 1 \\
\hline
\end{tabular}




\section{Architectural Design of ANN}

The basic architecture of feed-forward back-propagation based network is shown in Fig.4. The designed ANN is of MLP type consisting of 3 layers: input layer, tan-sigmoid hidden layer, and linear output layer. Other than input layer, each of the layers has a weight matrix $\mathrm{W}$, a bias vector $\mathrm{b}$ and an output vector a. The weight matrices connected to inputs are called input weights (IW) and weight matrices connected to the hidden layer outputs are called layer weights (LW). Additionally, superscripts are used to denote the source (second index) and the destination (first index) for the various weights, biases, and other elements of the network. $\mathrm{P}$ is the input vector, $\mathrm{n}$ is the layer output before transfer function and $\mathrm{a}$ is the actual output vector of a layer.

For optimized condition, the ANN was found to perform well if it was designed with 7 inputs, 10 tan-sigmoid neurons in hidden layer and 4 linear neurons in the output layer. The Detail structural design for feed-forward condition is depicted in Fig.5. It is still under challenging task when it comes to decide and select a number of neurons in the hidden layer. A large number of hidden neurons may deteriorate the performance of the network. It essentially requires huge memory to store huge numbers of network variables and hence training becomes complicated. However, if a too small number of neurons is used in the hidden layer, the network cannot adjust the weights and biases properly during training, which results in overfitting. Overfitting makes the network excessively complex, thus the non-generalized network generates random error and provides very poor classification. Due to the lack of specific rule in finding the numbers of hidden neurons to obtain optimized performance of the network, the performance criteria for different ANN architecture have been analyzed to identify the numbers of neurons.

After designing the ANN with specified architecture and different hidden neurons, a suitable and efficient backpropagation training algorithm is required for the adjustment of synaptic weights and biases at different layers. The back- propagation training algorithm basically sets the weights and biases to minimize the performance (Mean Square Error MSE) by using the gradient of the performance function. It was mentioned earlier that the classification efficiency of ANN depended on the selection of proper feature set, network structure, and training algorithm.

For this research, both the algorithms, namely LevenbergMarquardt and scaled conjugate gradient, are applied to find out their applicability and performance in ANN. The numerical optimization techniques based LevenbergMarquardt is the fastest and powerful method for training of moderate-sized feed-forward neural networks. The scaled conjugated gradient algorithm gives the solution to choose nearly conjugate directions of search instead of calculating the Hessian matrix or performing a line search. Some of the steps are also taken into consideration to improve the network generalization and to avoid overfitting. This is done by dividing the training input data in a random manner; $70 \%$ for training, $15 \%$ for validation and $15 \%$ for testing. In addition to these, the numbers of data points in each training set are plenty enough to estimate the total numbers of parameters for different architecture of the network. As per the requirement of improving the network generalization, the early stopping method was also applied during training. Two early stopping criteria are used: one is total mean squared error, MSE $<=0.001$ and another is the number of maximum training iterations set to 1000 . For each of the training sessions, the weights and biases for input and hidden layers are saved and are utilized in the next training session iteratively until a satisfactory simulation result is obtained. This is the general process to speed up the training, thus advancing the network with improved performance and less training time consumption. With different input features and corresponding targets, the network has been trained repeatedly until it has achieved some performance criteria (acceptable tolerance, training time, epochs).

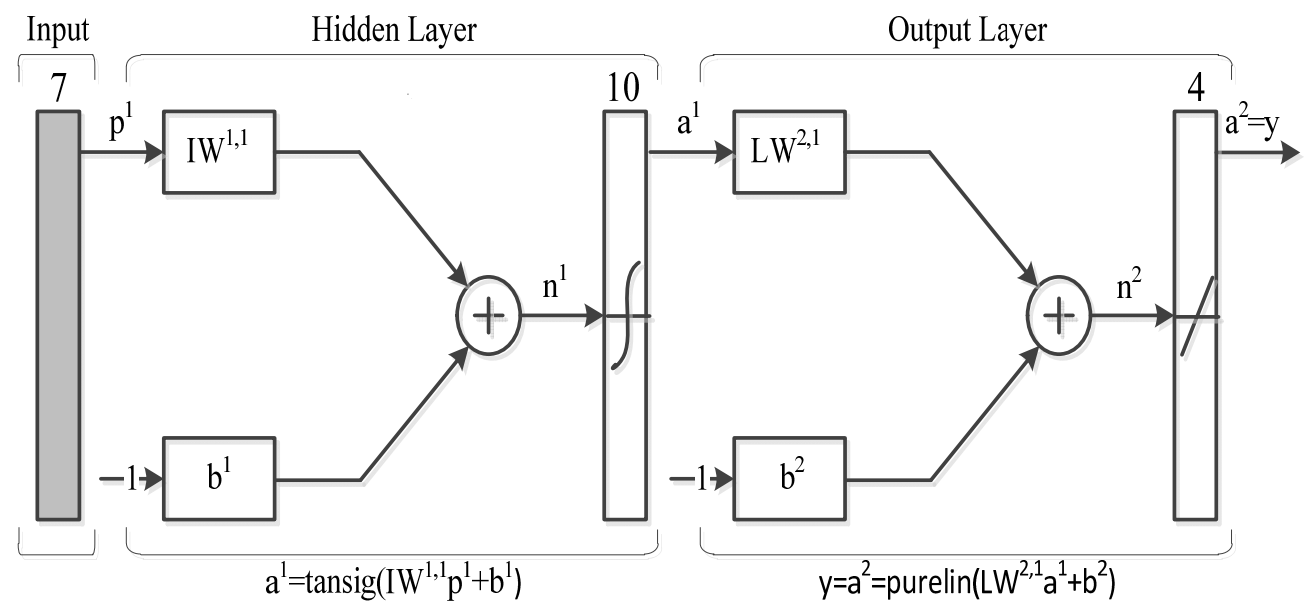

Fig.4. Architecture of Artificial Neural Network 


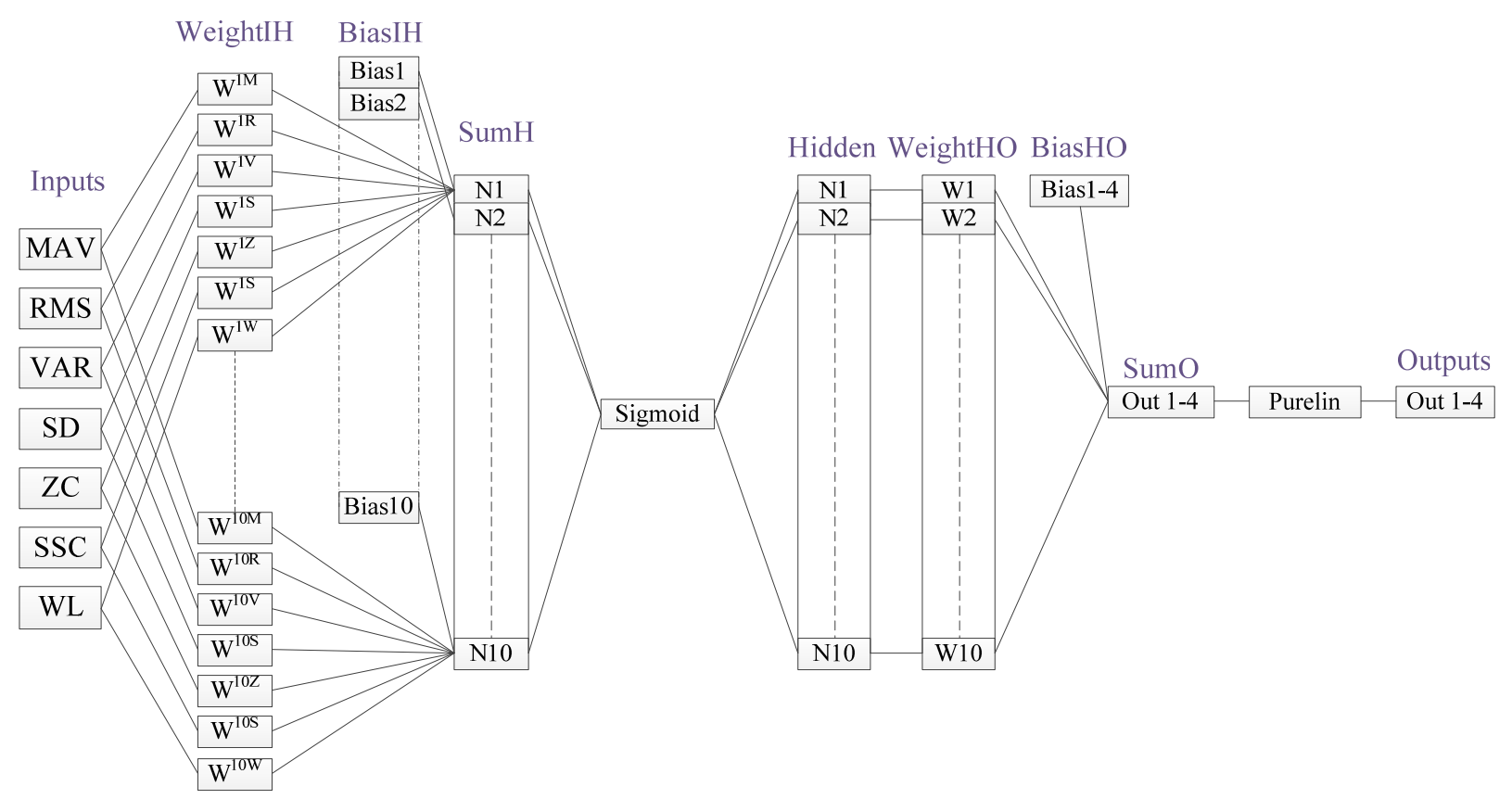

Fig.5. Detail Architecture view of Artificial Neural Network

\section{Optimizing the Neural Network for Classification}

The network has been trained with 204 sets of data points which are extracted from the denoised EMG signals for predefined hand movements. Each set of data comprises an input feature vector obtained from a specific type of hand movement and its corresponding output vector. The training and performance testing of ANN are done by applying both Levenberg-Marquardt (trainlm) and scale conjugate gradient (trainscg) algorithms. During each time of the training period, both algorithms function to adjust the weights and biases of the network in such a way as to minimize the MSE and hence increase the rate of network performance [14].

For the successful training, some of the conditions are set. These are the MSE set to 0.001, maximum validation failure set to 6 times, learning rate set to 0.05 , training status display set to 1 and the maximum number of epochs set to 1000. The performance of the training is evaluated with MSE of the training data, correlation coefficient, i.e. regression ( $R$ ) between the network outputs and corresponding target outputs and the characteristics of the training, validation, and testing errors. The networks with the best performance (lowest MSE, highest R) and almost similar error characteristics among the training, validation and testing are selected as the optimized network for the respective network architecture. During training session, if a large variation of error is being observed then the network structure could be considered unsatisfactory even though the MSE shows minimum value. Hence, further tuning and training of the network is necessary for achieving better performance. For the whole research work, different numbers of neurons for hidden layer have been chosen for both types of back-propagation training algorithm to find out most optimized and best performed network structure through studying the MSE and R.

\section{RESULTS}

The network responses and the performances for different architectural design are verified with the help of statistical analysis of MSE and R. Each of the different ANN architectures has been trained and then simulated for 10 times and their respective network performances (in terms of MSE and R) are collected. The graphical presentations of MSE are shown in Fig.6. where the comparison of the performances of different network structures can be viewed through error bars of mean and SD. The graphical presentation (Fig.7.) of the regression analysis of the network responses (R) for different network structures and different training algorithms is clearly promoting the ANN structure with 10 hidden neurons and Levenberg-Marquardt algorithm in preference to the other type.

The designed ANN structure has also been studied for different combinations of feature sets. Fig.8. shows the error histogram for different combinations of feature sets. Fig. 8 . (d) is clearly different from the other combinations. It has been found that, if the optimized ANN structure is fed with an input vector of seven features then maximum instances (around 550) of MSE errors are distributed near to zero line in the error histogram. 


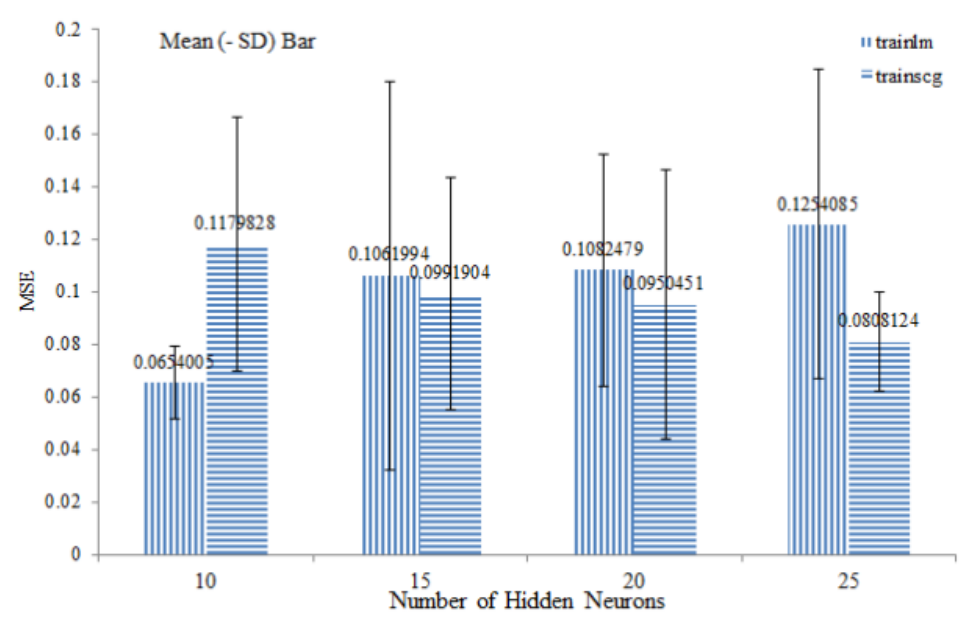

Fig.6. Mean-SD bar graph for MSE in between different ANN architecture

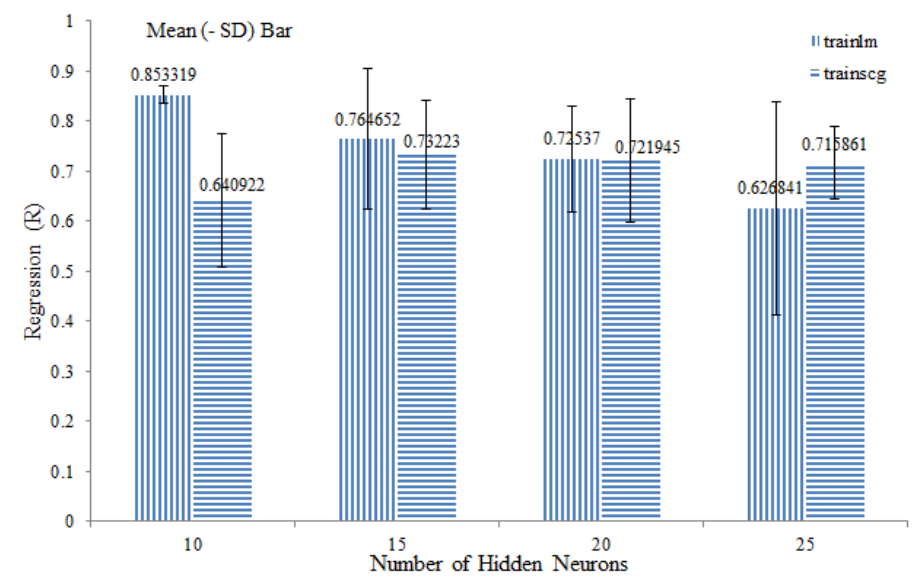

Fig.7. Mean-SD bar graph for Regression (R) in between different ANN architecture

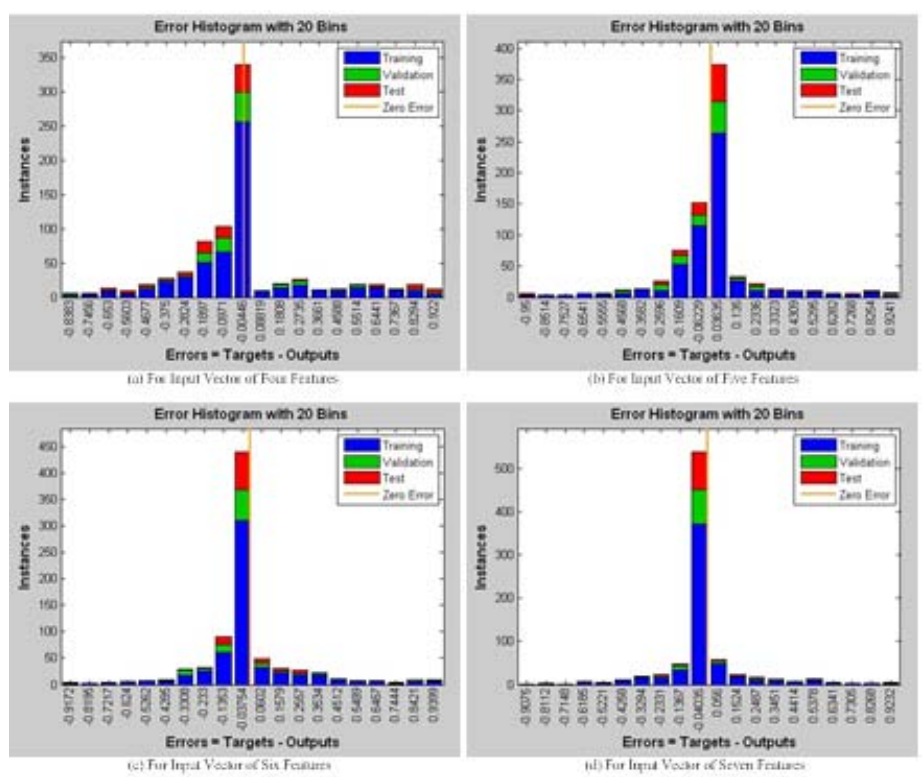

Fig.8. Error histogram for input vector of (a) four features, (b) five features, (c) six features and (d) seven features. 
The optimized ANN structure is trained by both types of learning algorithms with input-output feature vectors and is simulated for several times. For a single run, the training is stopped after 10 epochs since the validation error increased for more than six times as shown in Fig.9. The training, validation, and testing errors were in fairly good conditions with the characteristics set during training.

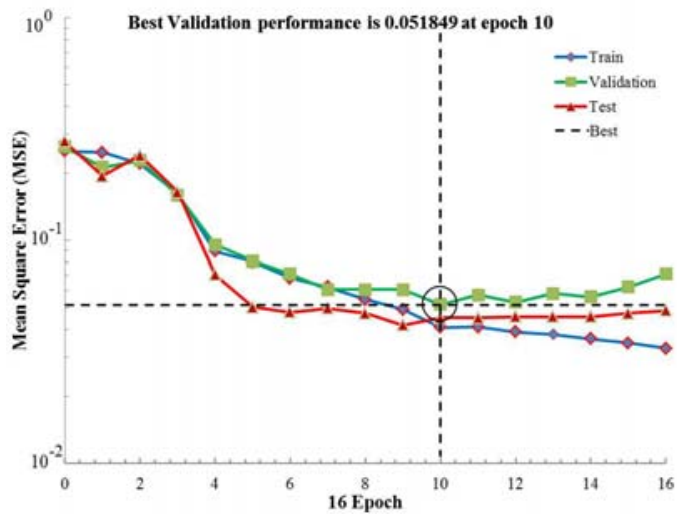

Fig.9. Training Validation and Test errors during training with Levenberg-Marquardt algorithm

For a single trial, the confusion matrix is also presented in Fig.10. with the detailed classification performance of the network during training, validation, testing and overall. The detailed performance of network during training, validation, testing and overall during a single trial is shown by confusion matrix for different classes in Fig.10. The numbers as presented with Target Class and Output Class are for different hand movements ('1'-Left, '2'-Right, '3'Up and ' 4 '-Down). Considering the Training Confusion Matrix, there are 142 hand movements in total (' 1 ' -37 , ' 2 '40, ' 3 '-35 and '4'-30), which are randomly chosen from input data to the neural network. The Light green color area is for correctly classified numbers; the red color area is for misclassified numbers, the deep green area for average classification, and blue color area is for total average classification. The percentage shows the ratio of the number with the total number of movements. If we consider a class ' 2 ', 39 numbers of movements are correctly classified. On the other hand is a single movement misclassified as class
' 3 ' which belongs to class ' 2 '. So, average classification for class ' 2 ' ' is $97.5 \%$ and $2.5 \%$ belongs to wrong classification. Finally, for Training Confusion Matrix, the total average classification by considering all types of class is $88.7 \%$ and $11.3 \%$ is a misclassification.

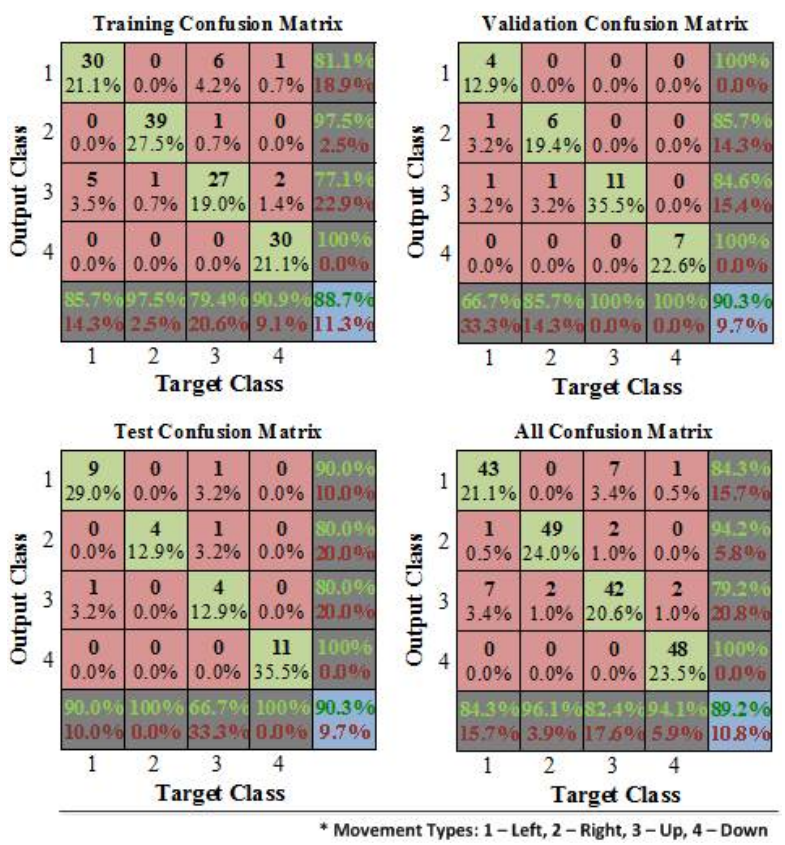

Fig.10. Confusion matrix for classification efficiency of the optimized structure of ANN

The generalization and performance of the trained ANN have also been tested for classifying completely unknown EMG signals. The extracted feature vectors for different hand motions are fed into the trained network without the corresponding target vectors. The probable output is to be expected as 1 in its index position for a specific type of hand movement. The classification output of the trained network is presented in Table 2., where p1, p2...p10 represent the extracted feature vectors from the test EMG signal. The bold numbers are denoted as the properly classified movements since these are the largest and/or closer to 1. Table 3 . presents the summary of classification performance of different ANN architecture.

Table 2. Test data as feature input vector and its corresponding classification output from trained ANN

\begin{tabular}{|c|c|c|c|c|c|c|c|c|c|c|}
\hline Input> & p1 & p2 & p3 & p4 & p5 & p6 & p7 & p8 & p9 & p10 \\
\hline \multirow{7}{*}{ 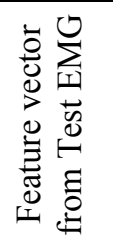 } & -0.12863 & -0.61608 & 0.37114 & 0.20342 & -0.13348 & -0.75516 & 0.22405 & 0.33569 & -0.24777 & -0.79584 \\
\hline & -0.12208 & -0.66515 & 0.34085 & 0.16422 & -0.22866 & -0.77763 & 0.09469 & 0.32470 & -0.34035 & -0.81355 \\
\hline & -0.51192 & -0.88580 & -0.00891 & -0.22084 & -0.60371 & -0.93406 & -0.29750 & -0.02931 & -0.69024 & -0.94736 \\
\hline & -0.12391 & -0.66707 & 0.33959 & 0.16281 & -0.23027 & -0.77885 & 0.09339 & 0.32338 & -0.34266 & -0.81470 \\
\hline & -0.17617 & -0.64118 & 0.19024 & 0.32336 & -0.13286 & -0.75488 & 0.09132 & 0.46075 & -0.34527 & -0.78546 \\
\hline & -0.29730 & 0.40541 & 0.10811 & -0.78378 & -0.05405 & 0.45946 & 0.29730 & -0.56757 & 0.08108 & 0.00000 \\
\hline & -0.42105 & -0.55263 & -0.65789 & 0.31579 & -0.23684 & 0.02632 & -0.52632 & 0.50000 & -0.55263 & -0.50000 \\
\hline \multirow{4}{*}{ 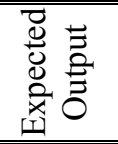 } & 0 & 0 & 1 & 0 & 0 & 0 & 1 & 0 & 0 & 0 \\
\hline & 0 & 0 & 0 & $\mathrm{~S} 1$ & 0 & 0 & 0 & 1 & 0 & 0 \\
\hline & 1 & 0 & 0 & 0 & 1 & 0 & 0 & 0 & 1 & 0 \\
\hline & 0 & 1 & 0 & 0 & 0 & 1 & 0 & 0 & 0 & 1 \\
\hline \multirow{4}{*}{ 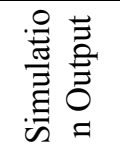 } & 0.0036 & 0.0004 & 0.6597 & 0.0081 & 0.0447 & 0.0024 & 0.6676 & 0.0273 & 0.0063 & 0 \\
\hline & 0.0085 & 0.0036 & 0.0032 & 0.955 & 0.0021 & 0 & 0.0017 & 0.8774 & 0.0003 & 0.0133 \\
\hline & 0.9928 & 0.0001 & 0.3271 & 0.091 & 0.7688 & 0.0018 & 0.5993 & 0.1906 & 0.8913 & 0.0008 \\
\hline & 0.0548 & 0.9991 & 0.02 & 0 & 0.1496 & 0.998 & 0.0093 & 0 & 0.288 & 0.9995 \\
\hline
\end{tabular}


MEASUREMENT SCIENCE REVIEW, Volume 13, No. 3, 2013

Table 3. Comparison and summary of classification performance for different ANN architecture

\begin{tabular}{|c|c|c|c|c|c|c|c|c|}
\hline \multirow{2}{*}{$\begin{array}{l}\text { Training } \\
\text { Function }\end{array}$} & \multirow{2}{*}{$\begin{array}{c}\text { Stop } \\
\text { Epochs }\end{array}$} & \multirow{2}{*}{$\begin{array}{c}\text { Regre- } \\
\text { ssion }\end{array}$} & \multirow{2}{*}{$\begin{array}{c}\text { Time } \\
\text { Elapsed }\end{array}$} & \multicolumn{4}{|c|}{ Classification Rate } & \multirow{2}{*}{$\begin{array}{l}\text { Hidden } \\
\text { Neurons }\end{array}$} \\
\hline & & & & Training & Validation & Test & Overall & \\
\hline \multirow{11}{*}{ 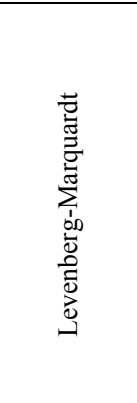 } & 15 & 0.8597 & 1.047 & 88.6 & 83.3 & 90 & 88 & \multirow{4}{*}{10} \\
\hline & 18 & 0.87251 & 0.921 & 94.3 & 66.7 & 80 & 88 & \\
\hline & 16 & 0.87401 & 0.8721 & 88.7 & 90.3 & 90.3 & 89.2 & \\
\hline & Avg & 0.86874 & 0.947 & 90.533 & 80.1 & 86.767 & 88.4 & \\
\hline & 33 & 0.85706 & 2.797 & 91.4 & 70 & 83.3 & 87 & \multirow{4}{*}{20} \\
\hline & 14 & 0.85508 & 1.218 & 90 & 80 & 86.7 & 88 & \\
\hline & 12 & 0.84772 & 1.094 & 92.9 & 76.7 & 83.3 & 89 & \\
\hline & Avg & 0.853287 & 1.703 & 91.433 & 75.567 & 84.433 & 88 & \\
\hline & 16 & 0.86112 & 2.36 & 92.1 & 80 & 76.7 & 88 & \multirow{4}{*}{30} \\
\hline & 11 & 0.85018 & 1.703 & 91.4 & 90 & 73.3 & 88.5 & \\
\hline & 14 & 0.85102 & 2.125 & 89.3 & 76.7 & 83.3 & 86.5 & \\
\hline \multirow{13}{*}{ 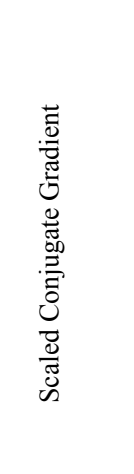 } & Avg & 0.854107 & 2.0627 & 90.933 & 82.233 & 77.767 & 87.667 & \\
\hline & 37 & 0.7839 & 0.703 & 80.7 & 83.3 & 83.3 & 82.43 & \multirow{4}{*}{10} \\
\hline & 27 & 0.7632 & 0.685 & 78.2 & 86 & 74.5 & 79.57 & \\
\hline & 32 & 0.7904 & 0.823 & 82.4 & 71.9 & 79.4 & 77.9 & \\
\hline & Avg & 0.77917 & 0.737 & 80.433 & 80.4 & 79.067 & 79.97 & \\
\hline & 31 & 0.802 & 0.797 & 78.6 & 90 & 82.7 & 83.77 & \multirow{4}{*}{20} \\
\hline & 35 & 0.8153 & 1.252 & 79 & 87.3 & 78.1 & 81.47 & \\
\hline & 34 & 0.79842 & 1.063 & 84.3 & 76.7 & 80 & 80.33 & \\
\hline & Avg & 0.80524 & 1.037 & 80.633 & 84.667 & 80.267 & 81.86 & \\
\hline & 34 & 0.80767 & 2.457 & 83.6 & 83.3 & 86.7 & 84.53 & \multirow{4}{*}{30} \\
\hline & 28 & 0.79215 & 1.073 & 81.2 & 72.1 & 69.5 & 74.27 & \\
\hline & 31 & 0.82531 & 1.352 & 86.6 & 76.5 & 78.8 & 80.63 & \\
\hline & Avg & 0.80837 & 1.627 & 83.8 & 73.3 & 78.333 & 79.81 & \\
\hline
\end{tabular}

\section{DISCUSSION}

Among various types of proposed ANN architecture, the feed-forward back-propagation type MLP is widely used to solve the pattern classification problems. The capability of learning from examples, the ability to reproduce arbitrary non-linear functions of input, and the highly parallel and regular structure of ANN make it especially suitable for pattern classification tasks [6], [27]. The ANN structures are capable of extracting useful information from the raw signal and can represent them through layers of significant numbers of neurons with associated weights and biases between the layers.

From Fig.6., the lowest mean and the lowest SD of the MSE have been observed in the ANN designed with 10 hidden neurons and trained with Levenberg-Marquardt training algorithm. In contrast to other network structures with different numbers of hidden neurons and scaled conjugate gradient training algorithm, this network performs better in terms of MSE. From Fig.7., it can easily be sorted out that the highest correlation coefficient is achieved for the ANN with 10 hidden neurons and Levenberg-Marquardt training algorithm. Compared to the other type of ANN architecture, this designed network shows the lowest mean and the lowest SD. It can be concluded that, for the efficient classification of single channel EMG signals, the ANN structure could be said to be optimal if it is designed with the Levenberg-Marquardt algorithm for back-propagation training and 10 neurons in its hidden layer. The MSE error histogram as presented in Fig.8. suggests that, using 7 features as input to the ANN will achieve better classification accuracy since most of the MSE errors for training are likely to concentrate near the "zero error" line.
The validity of the ANN training performance as presented in Fig.9. proves that the training, validation and test error curves are almost identical, which helps to converge the ANN more quickly by complying with the necessary characteristics set. By analyzing the classification performance in Table 3., it can be understood that the Levenberg-Marquardt algorithm based neural network with 10 hidden neurons yields the best classification rate and the required time is minimum. This network outperforms other network structures regarding the number of iterations required, time elapsed, classification rate, MSE and R. The performance of the Levenberg-Marquardt and scaled conjugate gradient training algorithms has been compared since these are well popular for faster training and solving pattern recognition/classification problems [13]. It is observed that the computational requirement of LevenbergMarquardt is a bit higher than the scaled conjugate gradient, however, it is actually responsible for providing better correlation and thus generates higher classification accuracy. Levenberg-Marquardt algorithm utilizes an adaptive learning rate and it does not show any oscillatory behavior during learning. The result analysis has clearly unfolded that Levenberg-Marquardt training algorithm with optimal network structure provides better classification with faster response, high error goal and less iteration which certainly agree with previous works [28], [29].

The classification performance of the designed ANN has also been compared with some of the previous researches as presented in Table 4. The study shows that the use of an increased number of channels increases the classification efficiency and another concluding remark from previous studies shows that the classification efficiency decreases 
with an increase in output class and vice versa [30]. From Table 4., with 2 channels EMG signals, 95\% and 93\% classification accuracy was achieved since there were only 2 classes and 4 classes, respectively. Use of EMG signals from multiple channels helps them to achieve a higher classification rate, whereas, since the number of classes increases for the second and fourth example, the classification performance reasonably decreases even if 4 channels of EMG signal are used. This research work utilizes single channel EMG signal and shows the success rate of ANN with Levenberg-Marquardt training algorithm. Single channel EMG signal classification has been improved compared to previous research works where multiple channels were utilized. It proves that a better classification performance can be achieved without any prior training to the subject.

Table 4. Classification performance comparison between different methods

\begin{tabular}{|ccccc|}
\hline Sl. & Method & $\begin{array}{c}\text { Number of } \\
\text { classes }\end{array}$ & $\begin{array}{c}\text { Number of } \\
\text { channels }\end{array}$ & $\begin{array}{c}\text { Classification } \\
\text { rate }\end{array}$ \\
\hline 1 & ANN with AR parameters [6] & 2 & 2 & $95 \%$ \\
\hline 2 & Neural Network [10] & 7 & 2 & $70 \%$ \\
\hline 3 & RBF and LVQ type Neural Network [17] & 4 & 2 & $93 \%$ \\
\hline 4 & LVQ type Neural Network [12] & 7 & 4 & $78 \%$ \\
\hline 5 & ANN with trainlm algorithm & 4 & single & $88.4 \%$ \\
\hline
\end{tabular}

\section{CONCLUSIONS}

The feed-forward ANN with back-propagation training algorithms has been trained with the extracted features from EMG signals to classify different hand motions. Different types of network architecture, depending on numbers of hidden neurons have been chosen and their classification performances have been analyzed. With thorough performance comparison and by tuning the network structure, it can be concluded that the ANN is optimized for better classification performance with 10 hidden neurons. The result shows that Levenberg-Marquardt based optimized ANN architecture can efficiently classify the single channel EMG signals with an average successful classification rate of $88.4 \%$. Moreover, the best overall classification performance, $89.2 \%$, has been achieved for a single trial. The comparison of simulation results also indicates that among the back-propagation learning algorithms, Levenberg-Marquardt algorithm performs better than the scaled conjugate gradient algorithm, which agrees with the previous study as mentioned earlier. The result significantly demonstrates the suitability of the proposed design and optimization process of ANN with LevenbergMarquardt algorithm for the classification of single channel EMG signal classification. However, the designed ANN structure has not yet been tested for the EMG signals from disabled or aged people. They could have different musculatures and different ways to move hand muscles which may result in huge noise and poor EMG signals. This may yield poor classification performance of designed ANN and it may require a redesign of the network through trial and error to achieve better classification performance.

\section{REFERENCES}

[1] Ahsan, M.R., Ibrahimy, M.I., Khalifa, O.O. (2009). EMG signal classification for human computer interaction: A review. European Journal of Scientific Research, 33 (3), 480-501.

[2] Reaz, M.B.I., Hussain, M.S., Mohd-Yasin, F. (2006). Techniques of EMG signal analysis: Detection, processing, classification and applications. Biological Procedures Online, 8 (1), 11-35.

[3] Ahsan, M.R., Ibrahimy, M.I., Khalifa, O.O. (2011). Neural network classifier for hand motion detection from EMG signal. In IFMBE Proceedings, Vol. 35. Springer, 536-541.

[4] Ahsan, M.R., Ibrahimy, M.I., Khalifa, O.O. (2010). Advances in electromyogram signal classification to improve the quality of life for the disabled and aged people. Journal of Computer Science, 6 (7), 705-715.

[5] Hiraiwa, A., Shimohara, K., Tokunaga, Y. (1989). EMG pattern analysis and classification by neural network. In IEEE International Conference on Systems, Man and Cybernetics, 14-17 November 1989. IEEE, 1113-1115.

[6] Putnam, W., Knapp, R.B. (1993). Real-time computer control using pattern recognition of the electromyogram. In EMBS 1993 : 15th Annual International Conference of the IEEE, 1236-1237.

[7] Naik, G.R, Kumar, D.K., Singh, V.P., Palaniswami, M. (2006). Hand gestures for HCI using ICA of EMG. In HCSNet Workshop on Use of Vision in HumanComputer Interaction (VisHCI '06), 1-3 November 2006. Australian Computer Society, 67-72.

[8] Englehart, K., Hudgins, B., Stevenson, M., Parker, P.A. (2002). A dynamic feedforward neural network for subset classification of myoelectric signal patterns. In EMBS 2002 : 17th Annual Conference of the IEEE, 20-25 September 1995. IEEE, Vol. 1, 819-820.

[9] Kelly, M.F., Parker, P.A., Scott, R.N. (2002). The application of neural networks to myoelectric signal analysis: A preliminary study. IEEE Transactions on Biomedical Engineering, 37 (3), 221-230.

[10] Itou, T., Terao, M., Nagata, J., Yoshida, M. (2001). Mouse cursor control system using EMG. In EMBS 2001 : 23rd Annual International Conference of the IEEE. Vol. 2, 1368-1369.

[11] Hudgins, B., Parker, P., Scott, R.N. (1993). A new strategy for multifunction myoelectric control. IEEE Transactions on Biomedical Engineering, 40 (1), 8294. 
[12] Jung, K.K., Kim, J.W., Lee, H.K., Chung, S.B., Eom, K.H. (2007). EMG pattern classification using spectral estimation and neural network. In Society of Instrument and Control Engineers (SICE 2007), 17-20 Sept. 2007. IEEE, 1108-1111.

[13] Hagan, M.T., Demuth, H.B., Beale, M.H. (1996). Neural Network Design. Boston, MA: PWS Publishing, 9.1-9.37.

[14] Ahsan, M.R., Ibrahimy, M.I., Khalifa, O.O. (2012). EMG motion pattern classification through design and optimization of neural network. In Internetional Conference on Biomedical Engineering (IcoBE 2012), 27-28 February 2012. IEEE, 175-179.

[15] Englehart, K., Hudgins, B. (2003). A robust, real-time control scheme for multifunction myoelectric control. IEEE Transactions on Biomedical Engineering, 50 (7), 848-854.

[16] Khezri, M., Jahed, M., Sadati, N. (2007). Neuro-fuzzy surface EMG pattern recognition for multifunctional hand prosthesis control. In IEEE International Symposium on Industrial Electronics, 4-7 June 2007. IEEE, 269-274.

[17] Tsenov, G., Zeghbib, A.H., Palis, F., Shoylev, N., Mladenov, V. (2007). Neural networks for online classification of hand and finger movements using surface EMG signals. In 8th Seminar on Neural Network Applications in Electrical Engineering (NEUREL 2006), 25-27 September 2006. IEEE, 167171.

[18] Phinyomark, A., Limsakul, C., Phukpattaranont, P. (2009). A novel feature extraction for robust EMG pattern recognition. Journal of Computing, 1 (1), 7180.

[19] Lyman, J., Freedy, A., Solomonow, M. (1977). System integration of pattern recognition, adaptive aided, upper limb prostheses. Mechanism and Machine Theory, 12 (5), 503-514.

[20] Naik, G.R., Kumar, D.K., Arjunan, S.P. (2010). Pattern classification of myoelectrical signal during different maximum voluntary contractions: A study using BSS techniques. Measurement Science Review, 10 (1), 1-6.

[21] Phinyomark, A., Limsakul, C., Phukpattaranont, P. (2011). Application of wavelet analysis in EMG feature extraction for pattern classification. Measurement Science Review, 11 (2), 45-52.
[22] Kim, J., Mastnik, S., André, E. (2008). EMG-based hand gesture recognition for realtime biosignal interfacing. In 13th International Conference on Intelligent User Interfaces (IUI '08), 13-16 January 2008. New York: ACM, 30-39.

[23] Ahsan, M.R., Ibrahimy, M.I., Khalifa, O.O. (2011). Electromyography (EMG) signal based hand gesture recognition using artificial neural network (ANN). In 4th International Conference on Mechatronics (ICOM 2011), 17-19 May 2011. IEEE, 1-6.

[24] Khezri, M., Jahed, M. (2007). A novel approach to recognize hand movements via sEMG patterns. In EMBS 2007 : 29th Annual International Conference of the IEEE, 23-26 August 2007. IEEE, 4907-4910.

[25] Cram, J.R., Criswell, E. (2010). Introduction to Surface Electromyography, 2nd ed. Sudbury, MA: Jones and Bartlett Publishers, 320-336.

[26] Hussain, M.S., Mamun, M. (2012). Effectiveness of the wavelet transform on the surface EMG to understand the muscle fatigue during walk. Measurement Science Review, 12 (1), 28-33.

[27] Subasi, A., Yilmaz, M., Ozcalik, H.R. (2006). Classification of EMG signals using wavelet neural network. Journal of Neuroscience Methods, 156 (1-2), 360-367.

[28] Zhao, J., Xie, Z., Jiang, L., Cai, H., Liu, H., Hirzinger, G. (2005). Levenberg-Marquardt based neural network control for a five-fingered prosthetic hand. In IEEE International Conference on Robotics and Automation, April 2005. IEEE, 4482-4487.

[29] Guo, X., Yu, H., Zhen, G., Yuliang, L., Yong, Z., Ying, Z. (2009). Artificial intelligent based human motion pattern recognition and prediction for the surface electromyographic signals. In International Conference on Information Technology and Computer Science (ITCS 2009), 25-26 July 2009. IEEE, 289-292.

[30] Naik, G.R., Kumar, D.K., Arjunan, S.P., Weghorn, H., Palaniswami, M. (2007). Limitations and applications of ICA in facial sEMG and hand gesture sEMG for human computer interaction. In 9th Biennial Conference of the Australian Pattern Recognition Society on Digital Image Computing Techniques and Applications, 3-5 December 2007. IEEE, 15-22.

Received August 13, 2012. Accepted June 10, 2013. 